\title{
Comment on 'How long is my toilet roll-a simple exercise in mathematical modelling'
}

\section{E. Arribas , I. Escobar \& R. Ramirez-Vazquez}

To cite this article: E. Arribas , I. Escobar \& R. Ramirez-Vazquez (2020): Comment on 'How long is my toilet roll-a simple exercise in mathematical modelling', International Journal of Mathematical Education in Science and Technology, DOI: 10.1080/0020739X.2020.1839682

To link to this article: https://doi.org/10.1080/0020739X.2020.1839682

\section{Published online: 04 Nov 2020.}

Submit your article to this journal $\sqsubset$

Q View related articles $₫$

View Crossmark data $\asymp$ 


\title{
Comment on 'How long is my toilet roll-a simple exercise in mathematical modelling'
}

\author{
E. Arribas (D) , I. Escobar (D) and R. Ramirez-Vazquez (D) \\ Applied Physics Department, Faculty of Computer Science Engineering, University of Castilla-La Mancha, \\ Albacete, Spain
}

\begin{abstract}
In the article 'How long is my toilet roll-a simple exercise in mathematical modelling' several models of increasing complexity are introduced and solved to calculate indirectly the length of paper on a toilet-roll. All these results are presented without errors. The authors of this comment believe the error analysis of measurements made in a laboratory is an important part of the experiment. The calculation of the absolute errors in indirect measurements provides us with information about the confidence that we must have in our experimental results. For that reason, the error calculation for all approximations has been made.
\end{abstract}

\section{ARTICLE HISTORY}

Received 16 May 2020

\section{KEYWORDS}

Paper roll; mathematical modelling; error analysis

\section{Introduction}

The error analysis of measurements made in a laboratory is an important part of the experiment. No measurement is free of error, even if all necessary precautions are taken into consideration (Taylor, 1996). Accidental errors are due to statistical fluctuations and alert us to the fact that every measurement must have its absolute error. It is a myth that something can be counted, measured, or weighed without making an error, repeating the process several times, as it is done by counting the ballots deposited in a ballot box. However, the best estimate of a scientific value obtained by measuring should always have an associated absolute error (Bevington \& Robinson, 2003; Drogs, 2007). This is an axiom from Physics Labs that we always use in our experiments. In these two articles (Arribas et al., 2015; Arribas et al., 2020) the exponent of the distance in a magnetic field of a dipole and a quadrupole is calculated in the laboratory and, in both cases, the exponents appear with their corresponding absolute errors. This is the purpose of this commentary.

In this article, Johnston (Johnston, 2013) calculates the length of paper on a toilet-roll in four ways using increasingly complex models. The toilet-roll has an internal radius $R$ and an external radius $R_{0}$, where $h$ is the width of a layer of paper. In this way, the number of turns the roll has is

$$
n=\frac{R_{0}-R}{h}
$$

CONTACT I.Escobar isabelmaria.escobar@uclm.es 
The numerical example studied in said work has the following values:

$$
\begin{aligned}
R_{0} & =55 \mathrm{~mm} \\
R & =20 \mathrm{~mm} \\
h & =0.36 \mathrm{~mm}
\end{aligned}
$$

The lengths obtained using the different four models are:

(1) Assuming that the roll consists of concentric annuli, each layer increasing in radius by $h$

$$
L_{1}=2 \pi R n+\pi n(n-1) h=22797 \mathrm{~mm}
$$

(2) Considering that the winding paper around a central spool is better approximated by a spiral

$$
L_{2}=2 \pi R n+\pi n^{2} h=22907 \mathrm{~mm}
$$

(3) Assuming that the paper is in a continuous sheet whereby it forms a spiral. Interestingly, the expression obtained here is the same as that obtained in the previous approximation

$$
L_{3}=2 \pi R n+\pi n^{2} h=\mathrm{L}_{2}
$$

Equation (7) can be written as (Thatcher, 1990)

$$
L_{3}=\frac{\pi}{h}\left(R_{0}^{2}-R^{2}\right)
$$

(4) The exact solution using the expression for arc length in polar coordinates (Zwillinger, 2003)

$$
L_{4}=2 \pi R n+\pi n^{2} h+\frac{h}{4 \pi} \ln \left(1+\frac{n h}{R}\right)=22907 \mathrm{~mm}
$$

considering the approximation $h / 2 \pi \ll R$. Observe that it is just the same numerical solution as in the previous approximation.

Finally, unrolling the toilet paper the length measured is

$$
L_{\text {unrolling }}=23200 \mathrm{~mm}
$$

All these results are presented without errors. But we believe no measure is free of error, even if all necessary precautions are taken into consideration. For that reason, we focused our work on the error calculation for the lengths obtained with the different approximations.

\section{Using error calculation}

Our comment is motivated by the necessity to consider absolute errors when experimental measurements are carried out.

In Equations (2) and (3) we suppose that the absolute error is given by ' 1 ' in the last significant figure. Thus,

$$
R_{0}=55 \pm 1 \mathrm{~mm}
$$




$$
R=20 \pm 1 \mathrm{~mm}
$$

Johnston (Johnston, 2013) comments that the thickness $\mathrm{h}$ of the paper was obtained by using a Vernier calliper and measuring the thickness of ten sheets of paper and then averaging. Thus, this magnitude with its error is given by

$$
h=0.360 \pm 0.002 \mathrm{~mm}
$$

assuming a Vernier calliper of sensitivity $0.02 \mathrm{~mm}$.

Firstly, we calculate the number of turns of paper from Equation (1) with its absolute error RMS (Berendsen, 2011), assuming that the correlation between the variables is null:

$$
\begin{aligned}
\varepsilon_{a}(n) & =\sqrt{\left(\frac{\partial n}{\partial R_{0}} \varepsilon_{a}\left(R_{0}\right)\right)^{2}+\left(\frac{\partial n}{\partial R} \varepsilon_{a}(R)\right)^{2}+\left(\frac{\partial n}{\partial h} \varepsilon_{a}(h)\right)^{2}} \\
& =\sqrt{\left(\frac{1}{h} \varepsilon_{a}\left(R_{0}\right)\right)^{2}+\left(\frac{-1}{h} \varepsilon_{a}(R)\right)^{2}+\left(\frac{-n}{h} \varepsilon_{a}(h)\right)^{2}}=4
\end{aligned}
$$

Thus,

$$
n=97 \pm 4
$$

Then, we will calculate the absolute error and relative error related to the paper lengths determined with the different approximations.

\subsection{The error related to the length $L_{1}$}

From Equation (5), we observe that the magnitude $L_{1}$ depends on three variables, $R, n$, and $h$ so we need three partial derivatives in order to calculate its absolute error

$$
\begin{aligned}
& \frac{\partial L_{1}}{\partial R}=2 \pi n \\
& \frac{\partial L_{1}}{\partial n}=2 \pi R+\pi h(2 n-1) \\
& \frac{\partial L_{1}}{\partial h}=\pi n(n-1) \\
& \varepsilon_{a}\left(L_{1}\right)=\sqrt{\left(\frac{\partial L_{1}}{\partial R} \varepsilon_{a}(R)\right)^{2}+\left(\frac{\partial L_{1}}{\partial n} \varepsilon_{a}(n)\right)^{2}+\left(\frac{\partial L_{1}}{\partial h} \varepsilon_{a}(h)\right)^{2}}=1500 \mathrm{~mm}
\end{aligned}
$$

Therefore, the length $L_{1}$ correctly expressed with its error is

$$
L_{1}=22800 \pm 1500 \mathrm{~mm}=22.8 \pm 1.5 \mathrm{~m}
$$

And the relative error of this measurement is

$$
\varepsilon_{r}\left(L_{1}\right)=\frac{1.5}{22.8}=0.07=7 \%
$$

Equation (20) indicates that the interval of allowed values for the length $L_{1}$ is

$$
L_{1} \in[21.3,24.3] \mathrm{m}
$$




\subsection{The error related to the lengths $L_{2}$ and $L_{3}$}

Under two different approximations, the same expression was obtained for lengths $L_{2}$ and $L_{3}$ (see Equation (7). From Equation (8), we observe that these lengths depend on three variables, $R_{0}, R$, and $h$ so we need three partial derivatives in order to calculate their absolute errors:

$$
\begin{aligned}
& \frac{\partial L_{3}}{\partial R_{0}}=\frac{2 \pi R_{0}}{h} \\
& \frac{\partial L_{3}}{\partial R}=\frac{-2 \pi R}{h} \\
& \frac{\partial L_{3}}{\partial h}=\frac{-L_{3}}{h} \\
& \varepsilon_{a}\left(L_{3}\right)=\sqrt{\left(\frac{\partial L_{3}}{\partial R_{0}} \varepsilon_{a}\left(R_{0}\right)\right)^{2}+\left(\frac{\partial L_{3}}{\partial R} \varepsilon_{a}(R)\right)^{2}+\left(\frac{\partial L_{3}}{\partial h} \varepsilon_{a}(h)\right)^{2}}=1000 \mathrm{~mm}
\end{aligned}
$$

Therefore, the lengths $L_{2}$ and $L_{3}$ correctly expressed with their error are

$$
L_{2}=L_{3}=22900 \pm 1000 \mathrm{~mm}=22.9 \pm 1.0 \mathrm{~m}
$$

being their relative errors significantly less than the previous one

$$
\varepsilon_{r}\left(L_{2}\right)=\varepsilon_{r}\left(L_{3}\right)=\frac{1.0}{22.9}=0.04=4 \%
$$

Equation (27) indicates that the interval of allowed values for the lengths $L_{2}$ and $L_{3}$ is

$$
L_{2}=L_{3} \in[21.9,23.9] \mathrm{m}
$$

which is within the range of values for $L_{1}$, see Equation (22).

\subsection{The error related to the length $L_{4}$}

From Equation (9), we observe that the magnitude $L_{4}$ depends on three variables, $R, n$, and $h$ so we need three partial derivatives in order to calculate its absolute error:

$$
\begin{aligned}
& \frac{\partial L_{4}}{\partial R}=2 \pi n-\frac{n h^{2}}{4 \pi R^{2}\left(1+\frac{n h}{R}\right)} \\
& \frac{\partial L_{4}}{\partial n}=2 \pi(R+n h)+\frac{h^{2}}{4 \pi R\left(1+\frac{n h}{R}\right)} \\
& \frac{\partial L_{4}}{\partial h}=\pi n^{2}+\frac{\ln \left(1+\frac{n h}{R}\right)}{4 \pi}+\frac{h n}{4 \pi R\left(1+\frac{n h}{R}\right)} \\
& \varepsilon_{a}\left(L_{4}\right)=\sqrt{\left(\frac{\partial L_{4}}{\partial R} \varepsilon_{a}(R)\right)^{2}+\left(\frac{\partial L_{4}}{\partial n} \varepsilon_{a}(n)\right)^{2}+\left(\frac{\partial L_{4}}{\partial h} \varepsilon_{a}(h)\right)^{2}}=1500 \mathrm{~mm}
\end{aligned}
$$


Therefore, the length $L_{4}$ correctly expressed with its error is

$$
L_{4}=22900 \pm 1500 \mathrm{~mm}=22.9 \pm 1.5 \mathrm{~m}
$$

being its relative error

$$
\varepsilon_{r}\left(L_{4}\right)=\frac{1.5}{22.9}=0.07=7 \%
$$

The interval of allowed values for the length $L_{4}$ is

$$
L_{4} \in[21.4,24.4] \mathrm{m}
$$

\section{Conclusions}

On the one hand, from Equations (22), (29), and (36) it is trivial to see that the four lengths calculated through four different models or approximations are compatible, within their respective error intervals, there is a common area to all of them.

On the other hand, we don't know how the length of the paper roll, $L_{\text {unrolling, }}$, has been measured directly to obtain the value shown in Equation (10). Nevertheless, the value of this magnitude, $L_{\text {unrolling }}=23.2 \mathrm{~m}$, is within the interval of allowed values for the lengths $L_{1}, L_{2}, L_{3}$, and $L_{4}$ calculated through four different approximations (see Equations (22), (29), and (36)). This means that all calculations and measurements are compatible.

Finally, if we focus on the results obtained for the varius lengths obtained with their respective approximations, Equations (20), (27), and (34), we observe that all of them have three significant figures. This is because the measurements made, Equations (11), (12), and (13), have two or three significant figures.

The calculation of the absolute errors in indirect measurements provides us with information about the confidence that we must have in our experimental results. No measurement is free of error, even if all necessary precautions are considered. By that, the error calculation for all approximations has been made.

\section{Disclosure statement}

No potential conflict of interest was reported by the authors.

\section{ORCID}

E. Arribas (i) http://orcid.org/0000-0002-0229-7923

I. Escobar (D) http://orcid.org/0000-0003-2927-8746

R. Ramirez-Vazquez (D) http://orcid.org/0000-0002-4773-4146

\section{References}

Arribas, E., Escobar, I., Ramirez-Vazquez, R., Gonzalez-Rubio, J., \& Beléndez, A. (2020). Measuring the magnetic field created by a linear quadrupole. The Physics Teacher, 58(3), 182-185. https://doi.org/10.1119/1.5145411

Arribas, E., Escobar, I., Suarez, C. P., Najera, A., \& Belendez, A. (2015). Measurement of the magnetic field of small magnets with a smartphone: a very economical laboratory practice for introductory physics courses. European Journal of Physics, 36(6), 065002. https://doi.org/10.1088/0143-0807/36/6/065002 
Berendsen, H. J. C. (2011). A student's guide to data and error analysis. Cambridge University Press. Bevington, P. R., \& Robinson, D. K. (2003). Data reduction and error analysis for the physical sciences (3rd ed.). McGraw-Hill Higher Education.

Drogs, M. (2007). Dealing with uncertainties. A guide to error analysis. Springer-Verlag Berlin Heidelberg.

Johnston, P. R. (2013). How long is my toilet roll? - a simple exercise in mathematical modelling. International Journal of Mathematical Education in Science and Technology, 44(6), 938-950. https://doi.org/10.1080/0020739X.2013.790502

Taylor, J. R. (1996). An introduction to error analysis. The study of uncertainties in physical measurements (2nd ed.). University Science Books.

Thatcher, D. (1990). The length of a roll of toilet paper, in mathematical modelling: A source book of case studies (I. Huntley \& D. J. G. James, Eds., pp. 51-58), Oxford University Press, chap. 4.

Zwillinger, D. (2003). CRC Standar mathematical tables and formulae (31st ed). Chapman\&Hall/CRC Press LLC. 